PROCEEDINGS OF THE

AMERICAN MATHEMATICAL SOCIETY

Volume 103, Number 2, June 1988

\title{
MULTIPLE NONTRIVIAL SOLUTIONS OF SEMILINEAR ELLIPTIC EQUATIONS
}

\author{
NORIMICHI HIRANO
}

(Communicated by Walter Littman)

\begin{abstract}
We give a condition for a semilinear elliptic equation to have two nontrivial solutions. Our condition does not demand any differentiability of the nonlinear term.
\end{abstract}

1. Introduction. Let $\Omega \subset R^{n}$ be a bounded domain with a smooth boundary $\partial \Omega$ and $g: R \rightarrow R$ be a continuous mapping such that $g(0)=0$. We study the boundary value problem of the form

$$
\Delta u+g(u)=0 \quad \text { in } \Omega,\left.\quad u\right|_{\partial \Omega}=0 .
$$

Let $\lambda_{1}<\lambda_{2} \leq \cdots \leq \lambda_{k} \leq \cdots$ denote the eigenvalues of the selfadjoint realization in $L^{2}(\Omega)$ of $-\Delta$ with the boundary condition. In [2], Ambrosetti and Mancini proved that if $g \in C^{2}, s g^{\prime \prime}(s)>0$ for all $s \neq 0$ and

$$
\lambda_{k-1}<g^{\prime}(0)<\lambda_{k}<g( \pm \infty)=\lim _{s \rightarrow \pm \infty} g^{\prime}(s)<\lambda_{k+1}, \quad \text { for some } k \geq 1,
$$

then the problem (1) has exactly two nontrivial solutions. Recently, Ahmad [1] proved that if $g \in C^{1}$ and

$$
0<g^{\prime}(0)<\lambda_{1}<\lim _{|t| \rightarrow \infty} g(t) / t<\lambda_{2},
$$

then the problem (1) has at least two nontrivial solutions.

In the present paper, we consider the case

$$
\lambda_{k-1}<b_{*} \leq b^{*}<\lambda_{k}<a_{*} \leq a^{*}<\lambda_{k+1}, \text { for some } k \geq 1
$$

where $a^{*}=\sup _{t \neq 0} g(t) / t, a_{*}=\liminf _{|t| \rightarrow \infty} g(t) / t, b^{*}=\limsup _{|t| \rightarrow 0} g(t) / t$, and $b_{*}=\inf _{t \in R} g(t) / t$. In assumption (4), we have implicitly supposed $\lambda_{k}$ is single. Our method is similar to that employed in [5], and does not demand that $g$ is differentiable or $\lim _{t \rightarrow \pm \infty} g(t) / t$ exists.

THEOREM. If (4) is satisfied, then the problem (1.1) has at least two nontrivial solutions.

REMARK. Our result is a partial extension of Theorem 1.2 of [2] and also Theorem 1 of [1]. In fact, it is easy to see that (4) holds if $s g^{\prime \prime}(s)>0$ for all $s \neq 0$ and $g$ satisfies (2). It is also obvious that if (3) holds and $0<g(t) / t<\lambda_{2}$ for $t \neq 0$, then (4) is satisfied with $k=1$ and $\lambda_{0}=0$. Our argument can be applied to a more general situation (e.g., $-\Delta$ can be replaced by a more general elliptic operator).

Received by the editors March 17, 1987.

1980 Mathematics Subject Classification (1985 Revision). Primary 35J25, 25J60; Secondary $47 \mathrm{~J} 15$.

Key words and phrases. Pseudo-monotone mapping, multiple solutions. 
2. Proof of Theorem. In the following, we write $L^{2}, H_{0}^{1}$ and $H^{-1}$ instead of $L^{2}(\Omega), H_{0}^{1}(\Omega)$ and $H^{-1}(\Omega)$, respectively. We denote by $\|\cdot\|,|\cdot|$ the norms of $H_{0}^{1}$ and $L^{2}$, respectively, the pairing between $H_{0}^{1}$ and $H^{-1}$ is denoted by $\langle\cdot, \cdot\rangle$. Let $H_{1}, H_{2}$ and $H_{3}$ be the subspaces of $L^{2}$ spanned by the eigenspaces corresponding to the eigenvalues $\left\{\lambda_{k+1}, \lambda_{k+2}, \ldots\right\},\left\{\lambda_{k}\right\}$, and $\left\{\lambda_{1}, \lambda_{2}, \ldots, \lambda_{k-1}\right\}$, respectively. Then $H_{1}, H_{2}$ and $H_{3}$ are orthogonal in $L^{2}$. Let $\phi$ be a normalized eigenfunction corresponding to $\lambda_{k}$. Then $\phi \in L^{\infty}(\Omega)$ and $H_{2}=\{k \phi: k \in R\}$. We denote by $P_{1}$, $P_{2}$ and $P_{3}$ the projections from $L^{2}$ onto $H_{1}, H_{2}$ and $H_{3}$, respectively. Suppose that $g$ satisfies the condition (4). Then there exist positive constants $\alpha, \beta, \rho$, and $\delta$ such that

$$
\alpha>\lambda_{k}, \quad g(t) / t \geq \alpha \text { for all } t \text { with }|t| \geq \delta
$$

and

$$
\beta<\lambda_{k}, \quad g(t) / t \leq \beta \text { for all } t \text { with } 0<|t| \leq \rho .
$$

Let $L=-\Delta$. For each $u, v \in H_{0}^{1}$, we set

$$
\langle T u, v\rangle=\left\langle L\left(u-2\left(P_{2}+P_{3}\right) u\right)-g\left(u-2\left(P_{2}+P_{3}\right) u\right), v\right\rangle .
$$

Then we can see that $u-2\left(P_{2}+P_{3}\right) u$ is a solution of (1) if and only if $T u=0$. So we will show the existence of $u \in H_{0}^{1}$ satisfying $T u=0$ by making use of an existence result for pseudo-monotone mappings. Let $K$ be a closed convex subset of a reflexive Banach space $E$. We denote by $\partial K$ and int $K$ the sets of boundary points and interior points of $K$, respectively. Let $T$ be a mapping from $K$ into the dual space $E^{\prime}$ of $E$. Then $T$ is said to be pseudo-monotone if $T$ satisfies the following condition:

If $\left\{u_{n}\right\} \subset K$ is a sequence such that $u_{n}$ converges weakly to $u$ and $\lim \sup \left\langle T u_{n}, u_{n}-u\right\rangle \leq 0$, then $(T u, u-z) \leq \liminf \left(T u_{n}, u_{n}-z\right)$ for each $z \in K$.

The following result is crucial for our argument.

THEOREM A. Let $K$ be a closed convex subset of $E$ with nonempty interior, and $T: K \rightarrow E^{\prime}$ be a pseudo-monotone mapping such that

(P) for each $z \in \partial K$, there exists $x \in \operatorname{int} K$ such that

$$
\langle T z, z-x\rangle \geq 0 \text {. }
$$

Then there exists $x_{0} \in K$ such that $T x_{0}=0$.

Theorem A is a simple version of Theorem 7.8 of Browder [3] (see also Theorem 0 of [5]). It can be proved by the same argument as in the proof of Theorem 1 of [4], so we omit the proof.

To apply Theorem A, we need the following three lemmas.

LEMMA 1 (CF. [5]). The mapping $T: H_{0}^{1} \rightarrow H^{-1}$ is pseudo-monotone.

PROOF. Let $\left\{u_{n}\right\} \subset H_{0}^{1}$ be a sequence such that $u_{n}$ converges to $u$ weakly in $H_{0}^{1}$ and

$$
\begin{aligned}
& \lim \sup \left\langle T u_{n}, u_{n}-u\right\rangle \\
& \quad=\lim \sup \left\langle L\left(u_{n}-2\left(P_{2}+P_{3}\right) u_{n}\right)-g\left(u_{n}-2\left(P_{2}+P_{3}\right) u_{n}\right), u_{n}-u\right\rangle \\
& \quad \leq 0
\end{aligned}
$$


Since $H_{0}^{1}$ is compactly embedded in $L^{2}$, we have that $u_{n}$ converges to $u$ strongly in $L^{2}$. We also have that $\left(P_{2}+P_{3}\right) u_{n}$ converges to $\left(P_{2}+P_{3}\right) u$ strongly in $L^{2}$. Then

$$
\lim \left\langle 2 L\left(P_{2}+P_{3}\right) u_{n}+g\left(u_{n}-2\left(P_{2}+P_{3}\right) u_{n}\right), u_{n}-u\right\rangle=0
$$

and therefore the inequality (9) implies that $\lim \sup \left\langle L u_{n}, u_{n}-u\right\rangle \leq 0$. Then it follows that $L u_{n}$ converges to $L u$ weakly in $H^{-1}$ and that $\lim \left\langle L u_{n}, u_{n}\right\rangle=\langle L u, u\rangle$. Thus we find that $T u_{n}$ converges to $T u$ weakly in $H^{-1}$ and $\lim \left\langle T u_{n}, u_{n}\right\rangle=\langle T u, u\rangle$. Then we obtain that

$$
\langle T u, u-z\rangle=\lim \left\langle T u_{n}, u_{n}-z\right\rangle \quad \text { for each } z \in H_{0}^{1},
$$

and this completes the proof.

LEMMA 2. There exists $s>0$ such that

$$
\left\langle T u, u-2 P_{2} u\right\rangle \geq 0 \quad \text { for all } u \in H_{0}^{1} \text { with }\left\|P_{2} u\right\| \leq s .
$$

PrOOF. We first choose a positive number $c$ so small that

$$
\begin{gathered}
\min \left\{\frac{1}{2}\left(\lambda_{k+1}-a^{*}\right),\left(b_{*}-\lambda_{k-1}\right)\right\}(\rho-c)^{2} / 4-\left(a^{*}-\lambda_{k}\right) c^{2} \\
+\frac{1}{2}\left(\lambda_{k+1}-a^{*}\right) d^{2}-2 c\left(a^{*}-\beta\right) d>0
\end{gathered}
$$

for all $d \in R$. Since $\phi \in L^{\infty}$, we can choose $s>0$ such that $\sup _{x \in \Omega}|z(x)| \leq c$ for all $z \in H_{2}$ with $\|z\| \leq s$. Let $u \in H_{1}^{0}$ with $\left\|P_{2} u\right\| \leq s$. We set, for simplicity, $v=P_{1} u, w=P_{2} u z=P_{3} u$, and $\tilde{u}=v-w-z$. We also set $A=\{x \in \Omega:|\tilde{u}|>\rho\}$ and $B=\{x \in \Omega:|\tilde{u}| \leq \rho\}$. From the definition of $T$, we have

$$
\begin{aligned}
\left\langle T u, u-2 P_{2} u\right\rangle & =\langle L(v-w-z)-g(v-w-z), v-w+z\rangle \\
& \geq \lambda_{k+1}|v|^{2}+\lambda_{k}|w|^{2}-\lambda_{k-1}|z|^{2}-\int_{\Omega} g(\tilde{u})(v-w+z) d x .
\end{aligned}
$$

Let $x \in A$. Then since $|w(x)| \leq c$, we have that $\max \{|v(x)|,|z(x)|\}>(\rho-c) / 2$. If $|z(x)| \geq|(v-w)(x)|$, then noting that $\tilde{u}(x)(v-w+z)(x) \leq 0$ we find from (4) that

$$
-g(\tilde{u}(x))(v-w+z)(x) \geq b_{*}\left(|z(x)|^{2}-|(v-w)(x)|^{2}\right) .
$$

If $|z(x)|<|(v-w)(x)|$, then we have by (4) that

$$
-g(\tilde{u}(x))(v-w+z)(x) \geq a^{*}\left(|z(x)|^{2}-|(v-w)(x)|^{2}\right) .
$$

Then from the inequalities above, we find that

$$
\begin{aligned}
& \lambda_{k+1}|v(x)|^{2}+\lambda_{k}|w(x)|^{2}-\lambda_{k-1}|z(x)|^{2}-g(\tilde{u}(x))(v-w+z)(x) \\
& \geq \lambda_{k+1}|v(x)|^{2}+\lambda_{k}|w(x)|^{2}-\lambda_{k-1}|z(x)|^{2}+b_{*}|z(x)|^{2}-a^{*}|(v-w)(x)| \\
& \geq\left(\lambda_{k+1}-a^{*}\right)|v(x)|^{2}+\left(\lambda_{k}-a^{*}\right)|w(x)|^{2}+\left(b_{*}-\lambda_{k-1}\right)|z(x)|^{2} \\
&+2\left(a^{*}-\beta\right) w(x) v(x)+2 \beta w(x) v(x) \\
& \geq \min \left\{\frac{1}{2}\left(\lambda_{k+1}-a^{*}\right),\left(b_{*}-\lambda_{k-1}\right)\right\}(\rho-c)^{2} / 4-\left(a^{*}-\lambda_{k}\right) c^{2} \\
&+\left(\frac{1}{2}\left(\lambda_{k+1}-a^{*}\right)|v(x)|^{2}-2 c\left(a^{*}-\beta\right)|v(x)|\right)+2 \beta w(x) v(x) \\
& \geq 2 \beta w(x) v(x) .
\end{aligned}
$$

Let $x \in B$. Then we have from (4) and (6) that

$$
-g(\tilde{u}(x))(v-w+z)(x) \geq b_{*}|z(x)|^{2}-\beta|(v-w)(x)|^{2} .
$$


Then we find that

$$
\begin{aligned}
\lambda_{k+1}|v(x)|^{2}+\lambda_{k}|w(x)|^{2}-\lambda_{k-1}|z(x)|^{2}-g(\tilde{u}(x))(v-w+z)(x) \\
\geq \\
\quad\left(\lambda_{k+1}-\beta\right)|v(x)|^{2}+\left(\lambda_{k}-\beta\right)|w(x)|^{2} \\
\quad+\left(b_{*}-\lambda_{k-1}\right)|z(x)|^{2}+2 \beta w(x) v(x) \\
\geq 2 \beta w(x) v(x) .
\end{aligned}
$$

Then combining (10) with (11) and (13), we obtain that

$$
\langle T u, u\rangle \geq 2 \beta\left(\int_{A} w(x) v(x) d x+\int_{B} w(x) v(x) d x\right)=2 \beta\langle w, v\rangle=0,
$$

and this completes the proof.

LEMMA 3. There exists $r>0$ such that $r>2 s$ and $\langle T u, u\rangle \geq 0$ for all $u \in H_{0}^{1}$ with $\|u\| \geq r$.

Proof. Let $\lambda$ be a positive number such that $a^{*}<\lambda<\lambda_{k+1}$. Then we have that $M=\min _{t \in R}\left\{\left(\lambda-b_{*}\right) t^{2}-\left(\alpha-b_{*}\right)(t+\delta)^{2}\right\}>-\infty$. Also we have that there exists $\omega>0$ such that $\|v\|^{2}-\lambda|v|^{2} \geq \omega\|v\|^{2}$ for all $v \in H_{1}$. Let $u \in H_{0}^{1}$. Let $v, w, z$ and $\tilde{u}$ be as in Lemma 2. We put $A=\{x \in \Omega:|\tilde{u}(x)| \geq \delta\}, B=\{x \in \Omega|\tilde{u}(x)|<\delta$, $|v(x)|<|(w+z)(x)|\}$ and $C=\{x \in \Omega:|\tilde{u}(x)|<\delta,|v(x)| \geq|(w+z)(x)|\}$. Then we have

$$
\langle T u, u\rangle \geq\|v\|^{2}-\lambda_{k}|w|^{2}-\lambda_{k-1}|z|^{2}-\int_{\Omega} g(\tilde{u}) u d x .
$$

From the conditions (4) and (5) we find that for each $x \in A$,

$$
-g(\tilde{u}(x)) u(x) \geq \alpha|(w+z)(x)|^{2}-a^{*}|v(x)|^{2} .
$$

Also we have by (4) that for each $x \in C$,

$$
-g(\tilde{u}(x)) u(x) \geq a^{*}|(w+z)(x)|^{2}-a^{*}|v(x)|^{2} \geq \alpha|(w+z)(x)|^{2}-a^{*}|v(x)|^{2} .
$$

Then we obtain that for each $x \in A \cup C$,

$$
\begin{aligned}
& -\lambda_{k}|w(x)|^{2}-\lambda_{k-1}|z(x)|^{2}-g(\tilde{u}(x)) u(x) \\
& \quad \geq-a^{*}|v(x)|^{2}+\left(\alpha-\lambda_{k}\right)|w(x)|^{2}+\left(\alpha-\lambda_{k-1}\right)|z(x)|^{2}+2 \alpha w(x) z(x) .
\end{aligned}
$$

Let $x \in B$. Then we have from (4) that

$$
-g(\tilde{u}(x)) u(x) \geq b_{*}|(w+z)(x)|^{2}-b_{*}|v(x)|^{2} .
$$

Then it follows that

$$
\begin{aligned}
&-\lambda_{k}|w(x)|^{2}-\lambda_{k-1}|z(x)|^{2}-g(\tilde{u}(x)) u(x) \\
& \geq-b_{*}|v(x)|^{2}-\lambda_{k}|w(x)|^{2}-\lambda_{k-1}|z(x)|^{2}+b_{*}|(w+z)(x)|^{2} \\
& \geq-b_{*}|v(x)|^{2}+\left(\alpha-\lambda_{k}\right)|w(x)|^{2}+\left(\alpha-\lambda_{k-1}\right)|z(x)|^{2} \\
&+\left(b_{*}-\alpha\right)|(w+z)(x)|^{2}+2 \alpha w(x) z(x) \\
& \geq-\lambda|v(x)|^{2}+\left(\alpha-\lambda_{k}\right)|w(x)|^{2}+\left(\alpha-\lambda_{k-1}\right)|z(x)|^{2} \\
&+\left\{\left(\lambda-b_{*}\right)|v(x)|^{2}-\left(\alpha-b_{*}\right)|(w+z)(x)|^{2}\right\}+2 \alpha w(x) z(x) .
\end{aligned}
$$


Then since $|(w+z)(x)|<|v(x)|+\delta$, we find that

$$
\begin{aligned}
& -\lambda_{k}|w(x)|^{2}-\lambda_{k-1}|z(x)|^{2}-g(\tilde{u}(x)) u(x) \\
& \geq-\lambda|v(x)|^{2}+\left(\alpha-\lambda_{k}\right)|w(x)|^{2} \\
& \quad+\left(\alpha-\lambda_{k-1}\right)|z(x)|^{2}+M+2 \alpha w(x) z(x) .
\end{aligned}
$$

Thus combining (15) and (18) with (14), we obtain

$$
\begin{aligned}
\langle T u, u\rangle & \geq\|v\|^{2}-\lambda|v|^{2}+\left(\alpha-\lambda_{k}\right)|w|^{2}+\left(\alpha-\lambda_{k-1}\right)|z|^{2}+M|\Omega|+2 \alpha\langle w, z\rangle \\
& \geq \omega\|v\|^{2}+\left(\alpha-\lambda_{k}\right)|w|^{2}+\left(\alpha-\lambda_{k-1}\right)|z|^{2}-M|\Omega| .
\end{aligned}
$$

From the inequality above, we can see that there exists $r>0$ such that $\langle T u, u\rangle \geq 0$ for all $u \in H_{0}^{1}$ with $\|u\| \geq r$. This proves the result.

Now we set $S=\left\{v \in H_{1}+H_{3}:\|v\| \leq r\right\}, S_{1}=\{k \phi: s \leq k \leq r\}$, and $S_{2}=$ $\{k \phi:-r \leq k \leq-s\}$. Let $E=H_{0}^{1}$ and $K_{i}=S \times S_{i}(i=1,2)$. We will show that the condition (P) holds with $K$ replaced by $K_{i}(i=1,2)$. Suppose that $u \in \partial K_{1}$. Then $\left\|P_{2} u\right\|=s$ or $\|u\| \geq r$ holds. If $\left\|P_{2} u\right\|=s$, then we have by Lemma 2 that $\left\langle T u, u-2 P_{2} u\right\rangle \geq 0$. Since $s<2 s<r$, we find that $2 P_{2} u \in \operatorname{int} K_{1}$. If $\|u\| \geq r$ and $\left\|P_{2} u\right\|=d>s$, then by Lemma 3, we have that $\langle T u, u-\varepsilon u\rangle=(1-\varepsilon)\langle T u, u\rangle \geq 0$ for each $\varepsilon>0$ with $s / d<\varepsilon<1$. Since $\varepsilon\|u\|<\|u\|$ and $\varepsilon\left\|P_{2} u\right\|>s$, we have $\varepsilon u \in \operatorname{int} K_{1}$. Thus we have shown that $(\mathrm{P})$ is satisfied. Then by Theorem $\mathrm{A}$, there exists $u_{1} \in K_{1}$ such that $T u_{1}=0$. Similarly, we have that there exists $u_{2} \in K_{2}$ such that $T u_{2}=0$. Since $K_{1} \cap K_{2}=\varnothing, u_{1} \neq u_{2}$ and this completes the proof of the result.

\section{REFERENCES}

1. S. Ahmad, Multiple nontrivial solutions of resonant and nonresonant asymptotically linear problems, Proc. Amer. Math. Soc. 96 (1987), 405-409.

2. A. Ambrosetti and G. Mancini, Sharp nonuniqueness results for some nonlinear problems, Nonlinear Anal. 5 (1979), 635-645.

3. F. E. Browder, Nonlinear operators and nonlinear equations of evolution in Banach spaces, Proc. Sympos. Pure Math., vol. 18, Part 2, Amer. Math. Soc., Providence, R. I., 1976.

4. N. Hirano and W. Takahashi, Existence theorems on unbounded sets in Banach spaces, Proc. Amer. Math. Soc. 80 (1980), 647-650.

5. N. Hirano, Unbounded nonlinear perturbations of linear elliptic problems at resonance, J. Math. Anal. Appl. (to appear).

FACULTY OF ENGINEERING, YOKOHAMA NATIONAL UNIVERSITY, TOKIWADAI HODOGAYAKU, YOKOHAMA, JAPAN

Current address: Center for Mathematical Sciences, University of Wisconsin-Madison, Madison, Wisconsin 53705 